\title{
Vingt-cinq ans de sida
}

$\mathrm{L}$ e sida a couvé pendant des années en Afrique avant qu'on le reconnaisse aux États-Unis, en I98I, comme une épidémie chez les hommes homosexuels. Son origine n'est plus un mystère. Grâce à la science avancée du séquençage génétique et de l'analyse phylogénétique, on connait la troupe de singes à l'origine du VIH-I en Afrique de l'Ouest. On ne sait toujours pas comment le virus a pu évoluer de son foyer naturel chez les chimpanzés pour devenir une zoonose, mais on attribue la pandémie à des causes crédibles comme les suivantes : nombreuse population, surpopulation, guerres, migration, pauvreté, commerce du sexe, autres infections transmises sexuellement, voyages, injection de drogues illicites et médicales, voire même la pratique de la circoncision (ou de la non-circoncision) masculine dans le contexte de la transmission sexuelle. Après un quart de siècle, le sida n'est qu'une autre des sombres réalités de la vie et s'ajoute à la liste des maladies infectieuses qui font des millions de victimes chaque année.

Dans les pays occidentaux, la connaissance médicale et scientifique du VIH et du sida a progressé régulièrement. En 1985, des scientifiques rivaux de la France et des États-Unis avaient isolé et nommé le virus. Les Centers for Disease Control and Prevention des États-Unis ont démontré par l'épidémiologie que le VIH cause le sida. On a entrepris de protéger l'approvisionnement en sang en sélectionnant les donneurs et en effectuant des analyses, mais le processus n'a pu se dérouler assez rapidement pour prémunir tous les receveurs - dans plusieurs pays, en dépit des capacités techniques, l'incapacité logistique et les limites financières ont eu pour résultat que beaucoup de personnes ont été infectées par le VIH provenant de transfusions sanguines pratiquées au milieu des années I980 et sont mortes par la suite du sida. Les tribunaux ont décidé que le retard avait causé un préjudice et les répercussions légales de cette décision sont toujours en train de prendre forme.

Les pays occidentaux ont franchi d'autres étapes marquantes, par exemple en développant un traitement aux antimicrobiens pour la prévention des infections opportunistes. Créée par méthode empirique, la thérapie préventive a amélioré la santé et la vie des personnes vivant avec le sida bien des années avant qu'un traitement spécifique contre le VIH ne soit disponible. De I995 à 2000 , on a mis au point une série de nouveaux médicaments contre le VIH qui, utilisés ensemble, ont fait reculer de façon spectaculaire le taux de mortalité relié au sida dans les pays où l'on a vendu le médicament et fourni des soins de santé.

En dépit de ces merveilles médicales et scientifiques dans le monde occidental, le sida fait des millions de victimes chaque année dans les pays les plus pauvres et les plus durement frappés. L'Organisation mondiale de la santé et ONUSIDA ont fixé un objectif ambitieux, soit de fournir d'ici 5 ans un traitement aux antirétroviraux à 3 millions de personnes (ce qui représente moins d'un an de l'incidence globale de l'infection par le VIH). À la fin de 2005, on avait raté l'objectif d'environ 2 millions de personnes ${ }^{1}$. En dépit des possibilités médicales et techniques, les réalités logistiques, économiques et politiques brutales ont empêché les traitements contre le VIH d'atteindre $95 \%$ des personnes qui en ont besoin.

À cause du sida, dans les pays les plus pauvres et les plus durement frappés, l'infection par le VIH combinée à la tuberculose a cassé subitement une tendance à l'amélioration régulière de l'espérance de vie. Plus le taux de mortalité chez les jeunes adultes est élevé, plus il y a de millions d'enfants orphelins. Les coûts pour les personnes et les sociétés dépasseront tous ceux qu'entrainent les morts et les bouleversements causés par la guerre ou par toute autre épidémie. Ce que réserve l'avenir dans les pays les plus pauvres, ce sont des dizaines de millions d'orphelins dont le sort constituera un autre legs du sida.

Après 25 ans de sida, nous avons la capacité médicale de traiter efficacement l'infection par le VIH, mais non la capacité logistique ou la volonté de fournir des traitements ou suffisamment de soins médicaux là où le plus besoin le fait le plus sentir. Voilà qui est peut-être aussi injuste que dans le cas des personnes infectées par le VIH à la suite de transfusions de sang lorsqu'on avait les moyens techniques de les protéger, mais la question ne fera l'objet d'aucune décision d'un tribunal. Les engagements politiques et les progrès de la science nous fourniront peut-être l'insaisissable vaccin contre le VIH avant que nous atteignions le prochain jalon pratique : offrir les réussites courantes de la médecine aux personnes les plus pauvres et qui en ont le plus besoin, et non seulement dans les pays les plus pauvres.

Outre la mise au point et la distribution de soins médicaux de base et spécifiques au VIH, le défi, qui sera relevé avec succès ou non, consistera à s'occuper des millions d'orphelins du sida. Puissent les 25 prochaines années produire autant de réussites pratiques que nous en avons connues sur le plan scientifique au cours des 25 premières années du sida.

\section{William Cameron}

Professeur de médecine

Université d'Ottawa

Hôpital d'Ottawa

Ottawa (Ont.)

Intérêts concurrents : aucuns déclarés.

\section{RÉFÉRENCE}

I. Nemes MIB, Beaudoin J, Conway S, et al. Evaluation of WHO's contribution to "3 by 5 ": main report. Geneva: World Health Organization; 30 mars 2006. Disponible à : www.who.int/hiv/topics/me/3 by5evaluationreport.pdf (consulté le 5 juillet 2006). 